\title{
A Webconferência como Ferramenta de Apoio à Tutoria nos Cursos da Graduação CEDERJ - Uma Avaliação sobre o Programa de Capacitação e Difusão de Uso
}

\section{Mara Lúcia Gomes de Campos*¹, Verônica Zegur Maguela ${ }^{2}$, Claudia Horta ${ }^{3}$, Daniel Fábio Salvador ${ }^{4}$}

\footnotetext{
${ }^{1}$ Coordenadora de tutoria e Bolsista Pesquisadora, Departamento de Mídias Digitais / Fundação CECIERJ. R. da Ajuda no 5, $15^{\circ}$ andar Centro - Rio de Janeiro - RJ - Brasil. maracampos@CECIERJ.edu.br

${ }^{2}$ Designer Instrucional e Pesquisadora, Departamento de Mídias Digitais / Fundação CECIERJ. R. da Ajuda no 5, $15^{\circ}$ andar Centro - Rio de Janeiro - RJ - Brasil. vmaguela@CECIERJ.edu.br

${ }^{3}$ Coordenadora de TAPs e Bolsista Pesquisadora, Departamento de Mídias Digitais / Fundação CECIERJ. R. da Ajuda no 5, $15^{\circ}$ andar Centro - Rio de Janeiro - RJ - Brasil. hortalmeida@gmail.com

${ }^{4}$ Professor e Pesquisador, Departamento de Mídias Digitais / Fundação CECIERJ. R. da Ajuda no 5, $15^{\circ}$ andar Centro - Rio de Janeiro - RJ - Brasil. salvador@CECIERJ.edu.br
}

\section{Resumo}

Um dos desafios da educação a distância é levar os alunos à proximidade encontrada nos cursos presenciais. Para isso, a webconferência é usada nos cursos de graduação do CEDERJ como uma ferramenta colaborativa que permite a maior integração entre os atores do processo de ensino-aprendizagem. O objetivo deste estudo é avaliar a implementação dessa ferramenta em tais cursos e sua forma de aplicação através de coleta de dados de salas de webconferência e da aplicação de um questionário aos tutores e coordenadores de disciplina que utilizam a ferramenta. Os resultados mostram que o aumento do uso de webconferência ocorre com o aumento das capacitações. Conclui-se que é necessário capacitar todos os agentes envolvidos no processo de usabilidade da ferramenta bem como ampliar as estratégias de condução e metodologias de planejamento para as sessões de webconferência.

Palavras-chave: Webconferência, Graduação CEDERJ, Educação a distância, Videotutoria. 


\title{
Web Conferencing to Support Mentoring Tool in \\ Unndergraduate CEDERJ Courses - an Evaluation of the \\ Training Program and Broadcast Use
}

\begin{abstract}
One of the challenges of distance education is to provide students with the closeness they find in classroom courses. To do so, web conferencing is used in undergraduate courses of CEDERJ as a collaborative tool that allows for greater integration between the actors of the teaching learning process. The aim of this study is to evaluate the implementation of this tool in such courses and its application form, through data collection of web conferencing rooms and a questionnaire applied to tutors and course coordinators who make use of the tool. The results show that the amount of of web conferencing increaes as the amount of training increases; and that the available rooms are still being underused. We conclude that it is necessary to empower all agents involved in the process to use the tool, and to expand strategies of conducting and planning methodologies for web conferencing.
\end{abstract}

Keywords: Web conferencing, Graduation CEDERJ, Distance education, Video mentoring. 


\section{Introdução}

Um dos desafios da educação a distância é promover a aproximação entre os estudantes e estudantes e tutores/professores, que caracteriza os cursos presenciais. Segundo Palloff e Pratt (2002), o uso de ferramentas colaborativas e cooperativas permite que as interações entre estudantes e professores sejam incorporadas aos cursos on-line e tornem-se essenciais para uma aprendizagem baseada na interação.

Nos cursos da Graduação CEDERJ, uma das estratégias utilizadas para permitir essa interação é a utilização de uma ferramenta de webconferência que recebe o nome de "videotutoria" devido ao uso para encontros tutor-aluno através da ferramenta síncrona de webconferência disponibilizada pela RNP (Rede Nacional de Ensino e Pesquisa) e implantada através do software Adobe Connect.

A Transposição Midiática ${ }^{1}$ requer que o professor/tutor assuma papéis variados, durante uma reunião de webconferência, tais como: papéis pedagógicos, gerenciais, sociais e técnicos (Garonce, 2009). Nesse contexto, Garonce constata a necessidade da elaboração de um modelo de formação para professores que irão atuar com a webconferência para ações pedagógicas.

O Departamento de Mídias Digitais da Fundação CECIERJ possui um programa de capacitação que visa preparar professores e alunos para o uso da ferramenta.

Este trabalho objetiva avaliar a implementação dessa ferramenta nos cursos da Graduação CEDERJ, demonstrando como o programa de capacitação vem influenciando seu uso e indicando ações para promoção da difusão dessa tecnologia por maior percentual de disciplinas e cursos do consórcio.

A divulgação dessa pesquisa colabora fortemente para implantação do uso da webconferência em outros cursos de educação a distância de forma a promover boas práticas no uso das tecnologias para o ensino virtual.

As vantagens da ferramenta de videotutoria são claras e relatadas na literatura, tais como o contato visual em tempo real amenizando a distância física entre alunos, professores e tutores e possibilitando a interação entre os participantes. Essa utilização de mecanismos visuais de interação dos "sistemas de webconferência multimodal e multimídia podem favorecer que sentimentos de empatia e interações pessoais sejam mais intensamente encorajados, pois oferecem múltiplas possibilidades de interação" 
(Dotta, p. 3, 2012). Há também a possibilidade de uso de atividades variadas como quadro branco, bate-papo, imagens entre outros recursos. Com essas funcionalidades, o tutor pode estimular os alunos a opinarem, criticarem e construírem juntos conceitos e ideias. Contudo, essas possibilidades dependem de aspectos importantes, como, por exemplo, a capacitação do tutor e do aluno para lidar com a ferramenta bem como o uso pedagógico que é feito da mesma.

\section{Metodologia}

A metodologia utilizada neste trabalho consistiu em um levantamento de dados com objetivo diagnóstico dos agendamentos realizados na plataforma Moodle, nos cursos da graduação CEDERJ, e da aplicação de um questionário, com questões mistas, direcionado aos tutores e coordenadores de disciplina que já utilizaram a ferramenta, onde se objetivou verificar as dificuldades encontradas nas principais formas de sua utilização

O período de coleta de dados foi realizado tomando como referência os primeiros semestres dos anos de 2012, 2013 e 2014. Os dados quantitativos foram processados em forma de gráficos.

\section{Programa de Capacitação}

O programa de capacitação do Departamento de Mídias Digitais para a ferramenta de videotutoria é direcionado a todos os atores da Graduação CEDERJ. Os tutores participam de curso, onde um dos módulos é a apresentação da ferramenta de videotutoria com a realização de oficinas on-line. Os coordenadores de disciplinas participam de oficinas presenciais em suas respectivas universidades ou on-line, aos alunos são disponibilizados tutoriais para impressão e em forma de vídeos bem como o suporte por e-mail e telefone.

Ações como e-mails com sugestão de uso da ferramenta, demonstrações em seminários e encontros como o "Encontro de Formação e avaliação CEDERJ", realizado em Itaipava-RJ em 2013, são utilizadas como forma de divulgação. 
Após a capacitação é oferecido suporte permanente aos usuários através de telefone e e-mail do Serviço de Atendimento ao Usuário da plataforma Moodle/CEDERJ e também a disponibilização de equipe especializada para treinamentos individualizados on-line ou presenciais, de forma a consolidar os conhecimentos adquiridos na oficina. A possibilidade de acompanhamento das primeiras reuniões dos tutores e coordenadores com seus alunos também são possíveis, com objetivo de dar maior segurança na condução das primeiras reuniões, sendo necessário para isso que as solicitem através dos canais de comunicação disponibilizados.

Tutoriais apoiam as oficinas e cursos e são disponibilizados na plataforma da Graduação CEDERJ para consulta permanente. Esse material possui foco diferenciado aos alunos e professores de forma a atender necessidades específicas segundo o perfil de atuação na ferramenta: Host (Tutor ou Coordenador de disciplina - conduz a reunião) ou participante (aluno).

\section{Resultados e discussão}

Quanto aos objetivos das reuniões, o Gráfico 1 demonstra a forma de utilização da ferramenta pelos tutores e coordenadores de disciplina da Graduação com destaque para aulas de revisão para avaliações presenciais e a distância e plantão para dúvidas. Esses resultados demostram que o uso da ferramenta por tutores ainda tem foco em um perfil de tutoria reativa, para resolução de perguntas e alunos, e não a proposição ativa de dinâmicas e apresentações que promoveriam maior engajamento e aproveitamento do potencial colaborativo da ferramenta.

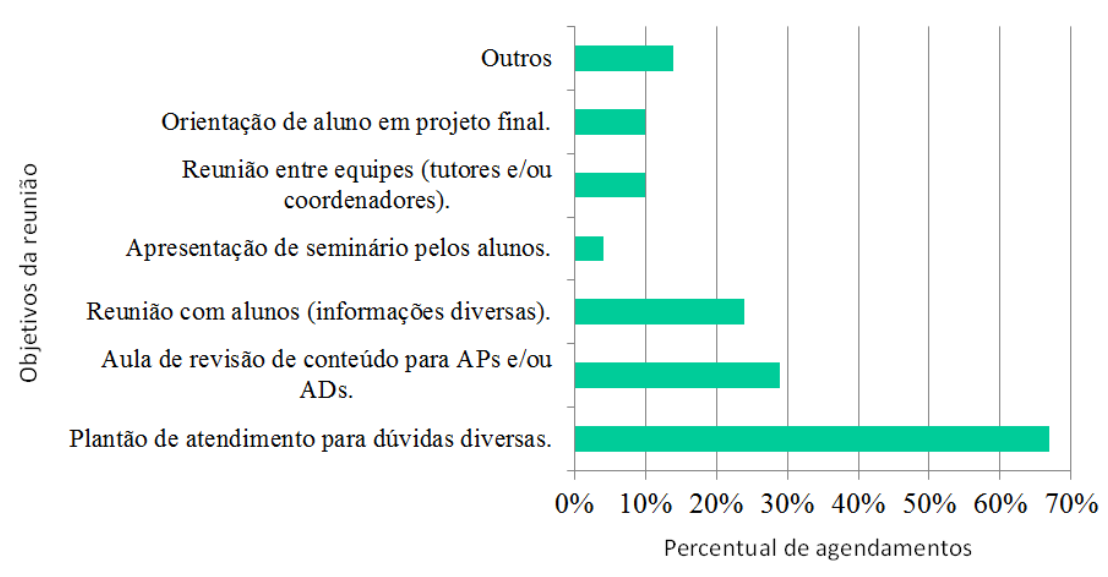

Gráfico 1: Objetivos das reuniões agendadas. 
No Gráfico 2, são apresentados os recursos utilizados nessas reuniões, onde constata-se que são, principalmente, envio de arquivos e compartilhamento de documentos. Fica evidente neste gráfico que muitos recursos não são explorados de forma significativa por uma grande parte dos usuários.

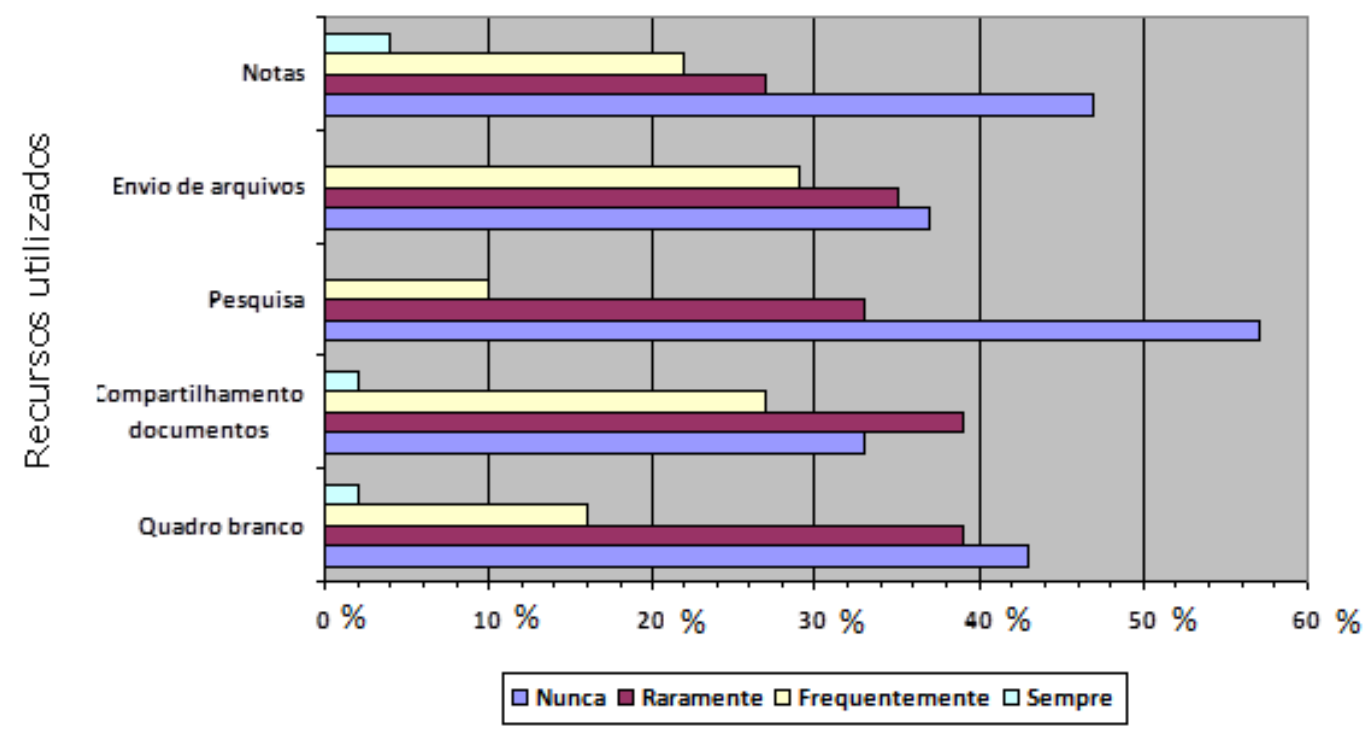

Porcentagem de Frequência de utilização

Gráfico 2: Recursos do Adobe Connect utilizados durante as reuniões.

A média de participantes nas reuniões é de até 10 pessoas - Gráfico 3, o que indica uma baixa participação dos alunos, considerando-se que as disciplinas da Graduação CEDERJ possuem em média 300 a 800 alunos para disciplinas de primeiro período com números decrescentes para demais períodos.

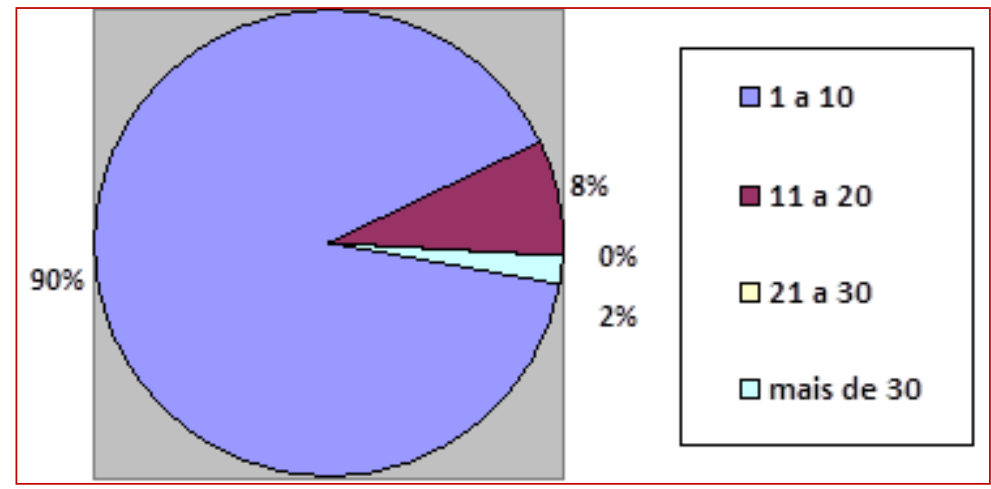

Gráfico 3: Quantidade média aproximada de participantes por reunião. 
O Gráfico 4 mostra o total de disciplinas que utilizaram a ferramenta de videotutoria nos primeiros semestres de cada ano. Constatou-se que a utilização da ferramenta aumentou ao longo do período analisado, porém em relação à quantidade de disciplinas que são oferecidas na Graduação CEDERJ, aproximadamente 600 disciplinas, podemos verificar que pouco mais de $10 \%$ estão usando o recurso. Foram consideradas apenas as disciplinas com mais de cinco agendamentos por semestre na computação dos dados.

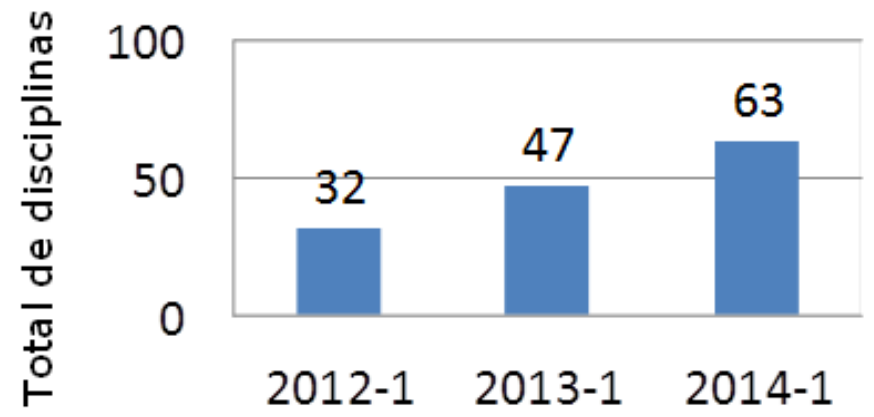

Gráfico 4: Total de disciplinas utilizando a videotutoria.

A Graduação CEDERJ oferece um total de 14 cursos, No Gráfico 5, estão apresentados os cursos que mais utilizaram a ferramenta no período analisado. Apenas $35 \%$ dos cursos estão utilizando a ferramenta de videotutoria, o que sugere a necessidade de aumentar as ações de capacitação e divulgação da ferramenta.

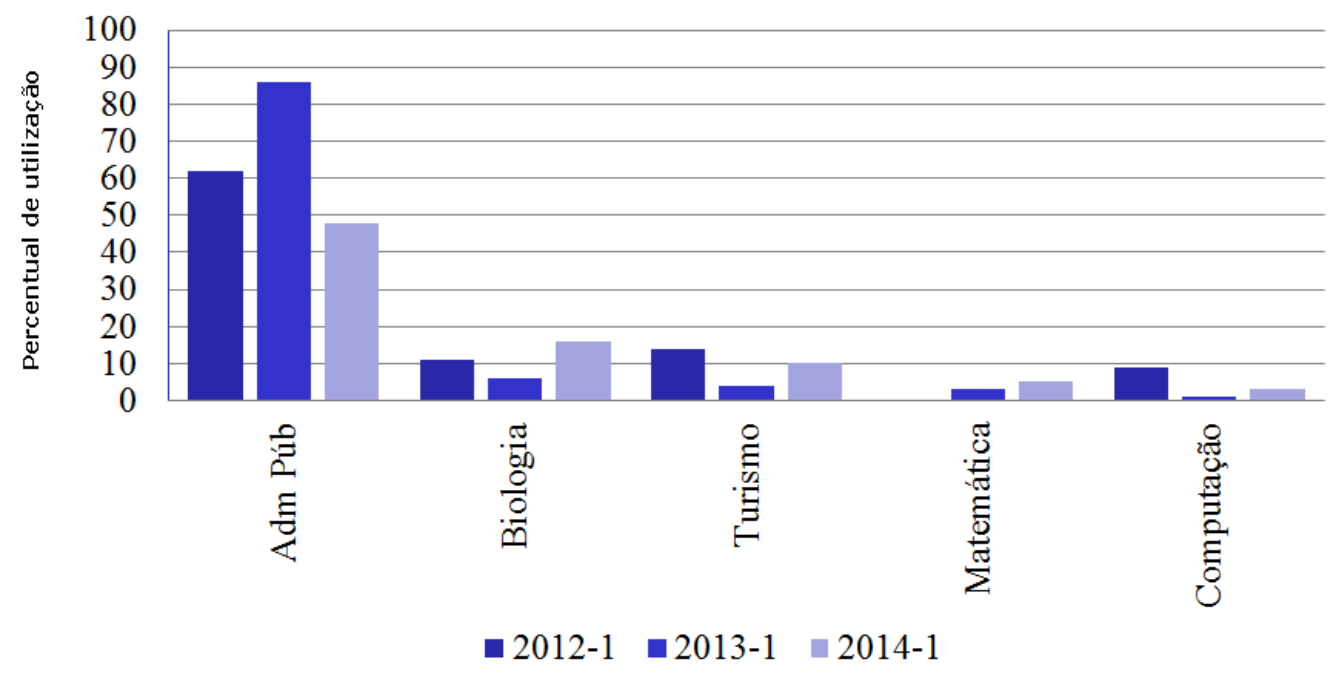

Cursos da Graduação com maior índice de participação

Gráfico 5: Porcentagem de reuniões por curso. 
Um fator técnico que poderia estar sendo limitante é que o sistema de agendamento oferecia, até o ano de 2013, somente uma hora de agendamento diário para cada disciplina. Isso foi alterado para quatro sessões diárias a partir de 2014.

O aumento do número de agendamentos diários por disciplina foi resultante da análise do Gráfico 6 e das questões abertas desse mesmo questionário, onde os tutores e coordenadores apontaram que o número de sessões diárias permitidas por disciplina era insuficiente para as ações pedagógicas desenvolvidas em algumas delas.

A duração de uma sessão de webconferência varia de acordo com o objetivo da aula, mas é importante o planejamento para que não ocorram problemas técnicos que interfiram no nível de interesse dos alunos (problemas de imagem e som) e que sejam dinâmicas introduzindo espaços para discussões que envolvam os alunos entremeando as exposições orais (Garonce 2009, p 187).

No Gráfico 6, podemos ver que para a maioria dos respondentes ao questionário aplicado, quando perguntados sobre o tempo de duração da reunião em relação ao agendamento para as sessões de videotutoria, o tempo de uma hora diária oferecido é suficiente, porém temos uma quantidade significativa que consideraram esse tempo ruim ou apenas razoável.

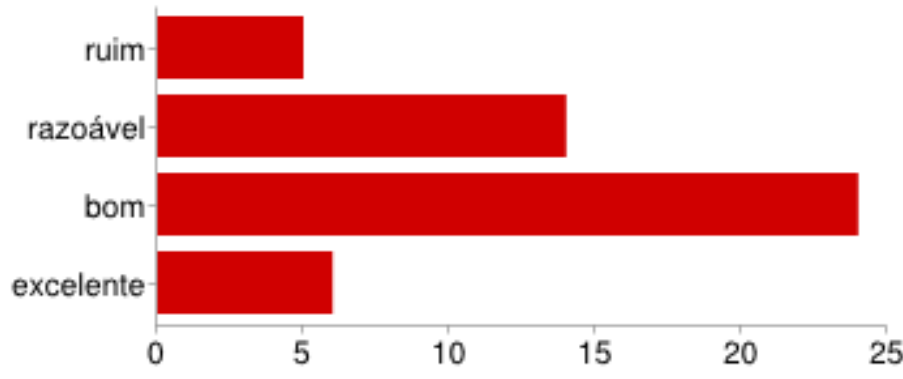

Quantidade de respondentes

Gráfico 6: Impressão dos respondentes ao questionário sobre o tempo de duração das reuniões em uma hora/dia oferecido às disciplinas do Consórcio CEDERJ.

Os resultados apresentados no Gráfico 7 demonstram que houve um aumento significativo na utilização da ferramenta entre o período de 2012 a 2014, coincidindo com o aumento nas capacitações e com a liberação para agendamento de um maior 
número de sessões de videotutoria por disciplina, implementada no primeiro semestre de 2014 (2014-1).

Durante o período analisado foram realizadas 28 oficinas e quatro cursos.

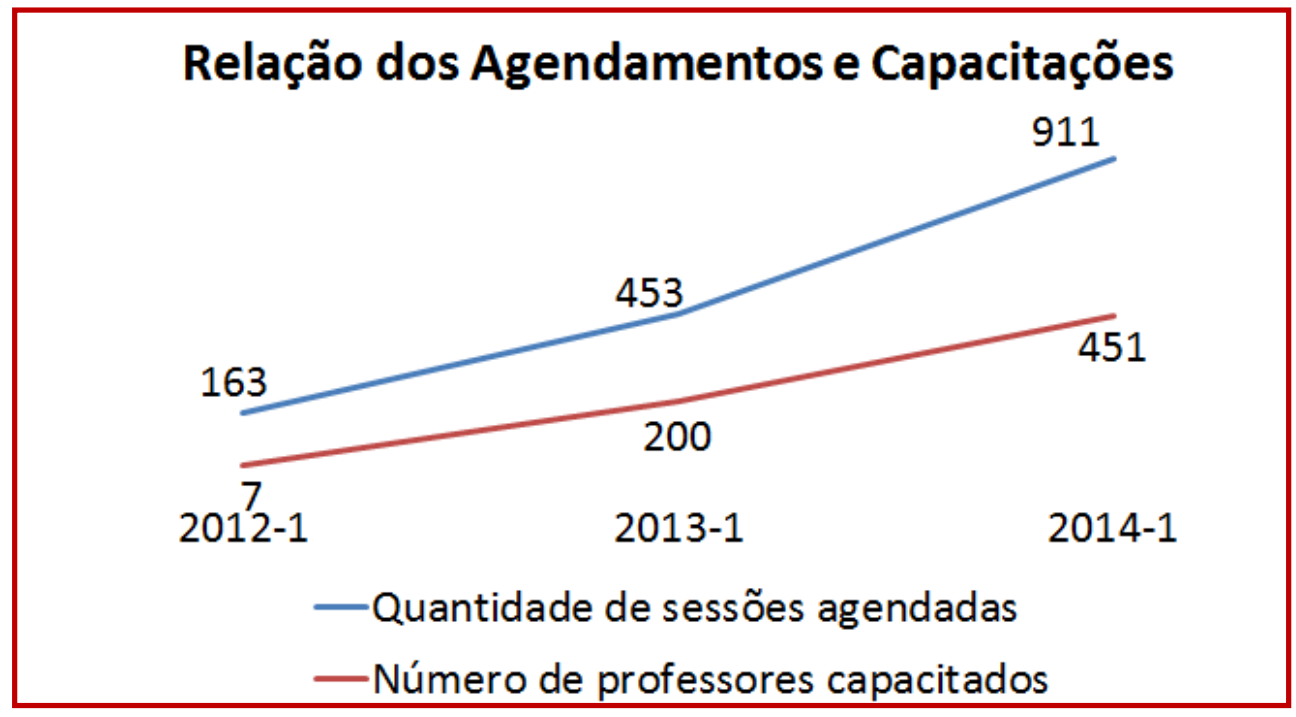

Gráfico 7: Relação dos agendamentos e capacitações realizados no período do primeiro semestre de 2012 (2012-1) ao primeiro semestre de 2014 (2014-1).

Os tutores e coordenadores que responderam ao questionário relataram como pontos fortes da ferramenta:

- Contato em tempo real com o aluno;

- Troca de arquivos de texto, imagens;

- Não depende de download de software para participação;

- Interação on-line;

- Possibilidade de demonstrar resolução de atividades em tempo real;

- Funcionalidades como quadro branco, bate-papo, notas;

- Maior aproximação tutor/aluno através da possibilidade de conhecê-lo visualmente;

- Excelente opção para orientação de monografia;

- Boa interface;

- Favorece a participação em reuniões e cursos de capacitação. 
E como pontos fracos:

- Instabilidade de conexão;

- Baixa participação dos alunos;

- Dificuldade para mediar muitos alunos;

- Problemas de conexão nos polos e universidades;

- Disponibilidade de tempo;

- Necessidade de suporte devido a sua complexidade.

Como sugestão, as respostas dos tutores e coordenadores de disciplina indicaram o seguinte:

- Estimular o uso por parte dos alunos e capacitá-los;

- Melhorar o sistema de agendamento para não haver conflito entre disciplinas;

- Uma oficina voltada para alunos cegos e surdos;

- Melhorar as conexões nas universidades e polos;

- Possibilidade de horário flexível.

\section{Conclusão}

Este trabalho demonstra que a capacitação e as ações realizadas se refletem no crescente aumento do agendamento para uso da ferramenta. Concordamos com Dotta (2012) que é necessário capacitar todos os agentes envolvidos no processo, incluindo estratégias de condução e metodologias de planejamento para essa modalidade. Com isso, o Departamento de Mídias Digitais mantém constantes as ações sobre esses agentes na Graduação CEDERJ e tem como próximos passos aumentar o foco de suas 
capacitações no uso das possibilidades pedagógicas da mesma, nos diversos conteúdos e áreas, além do uso técnico da ferramenta para maior confiança por parte dos tutores.

O fato de os tutores ainda relatarem que a ferramenta está sendo usada, predominantemente, para plantão de dúvidas sugere que o potencial colaborativo está sendo subutilizado e que ações sobre outras estratégias pedagógicas podem ser mais bem trabalhadas nas capacitações. Entretanto, o maior número de agendamentos e disciplinas que a estão utilizando demostram efeitos positivos do processo de capacitação e promoção do uso da webconferência em cursos a distância.

\section{Nota}

1 Processo de transposição de ações docentes do ambiente tradicional para o virtual com o estabelecimento da relação presencial conectada possibilitada pela webconferência (Garonce, 2009).

\section{Referências bibliográficas}

Palloff, R. M. \& Pratt, K. (2002). Construindo Comunidades de Aprendizagem no Ciberespaço: estratégias eficientes para a sala de aula on-line. Tradução: Vinicius Figueira, Porto Alegre: Artmed.

Dotta, S.; Braga, J. \& Pimentel, E. (2012). Condução de aulas síncronas em sistemas de webconferência multimodal e multimídia. Anais do $23^{\circ}$ Simpósio Brasileiro de Informática na Educação. ISSN 2316-6533 - Rio de Janeiro.

Disponível em http://www.lbd.dcc.ufmg.br/colecoes/sbie/2012/0015.pdf

Garonce, F. V. (2009). Os Papéis Docentes nas Situações de Webconferência: um estudo de caso acerca da ação educativa presencial conectada. Tese de doutorado - Faculdade de Educação, Universidade de Brasília, Distrito Federal.

Rede Nacional de Ensino e Pesquisa - RNP (n.d.). Acesso em 05 de dezembro de 2014 e disponível em http://www.rnp.br 